\title{
A Rare Case of Acute Renal Failure: Rhabdomyolysis Due to Hyperosmolar Hyperglycemic State
}

Tugrul Burak Genc, Yildiz Okuturlar, Hakan Kocoglu, Samet Sayilan, Yesim Ozdem Inan, Ezgi Erdogan, Meral Mert, Betul Erismis, Bahar Ozdemir, Ozlem Harmankaya, Abdulbaki Kumbasar

Bakirkoy Dr. Sadi Konuk Education and Research Hospital

\section{Introduction:}

Rhabdomyolysis is usually attributed to trauma. However, there is an association of rhabdomyolysis with hyperosmolar states. It is also important to realize that hyperosmolar coma can be the presenting complaint of a diabetic seeking medical attention for the first time. In this case-report we aimed to present a patient with acute kidney injury due to rhabdomyolysis and hyperosmolar state.

\section{Case Report:}

49-year-old black male patient was brought to emergency room because of new onset confusion during an international flight. His past medical history was remarkable for only asthma. Bibasilar decreased breath sounds and $2+$ bilateral pretibial edema were detected on physical examination. His laboratory studies were as follows: blood glucose $1505 \mathrm{mg} / \mathrm{dl}$, urea $163 \mathrm{mg} / \mathrm{dl}$, creatinine $9.8 \mathrm{mg} / \mathrm{dl}$, creatine-kinase $(\mathrm{CK}) 45,750 \mathrm{U} / \mathrm{L}$, sodium $(\mathrm{Na}) 146 \mathrm{mmol} / \mathrm{l}$, potassium $(\mathrm{K}+) 4.67$ $\mathrm{mmol} / \mathrm{l}$, blood $\mathrm{pH} 7.3$, osmolarity $368 \mathrm{mosm} / \mathrm{l}$. His urinary ultrasonography was normal. The patient was brought to intensive care unit and received continuous arteriovenous hemofiltration. His consciousness level gradually improved, his urinary output increased and creatinine level decreased to normal value. The patient was discharged after his blood glucose levels were controlled.

The exact mechanism of rhabdomyolysis in a hyperosmolar state remains unclear. Singhal et al. concluded that serum sodium, osmolality and glucose levels are the major determinants for the occurrence of rhabdomyolysis in the diabetic state. Pharmaceutical agents, alcohol, illicit drugs, hypokalemia, hypophosphatemia, hypothermia and hyperosmolar situations are significant causes of rhabdomyolysis. To our knowledge, there are around a dozen cases reported in the literature. Although it is rarely clinically important, it has been suggested that over $50 \%$ of patients presenting with hyperosmolar state may develop rhabdomyolysis to greater or lesser degree. 